\title{
WiRA: An Approach to Resource Control in WiMAX Systems
}

\author{
Eduardo Cerqueira ${ }^{1}$, Augusto Neto ${ }^{1}$, Marília Curado ${ }^{1}$, Paulo Mendes $^{2 \dagger}$, and Edmundo Monteiro ${ }^{1}$ \\ ${ }^{1}$ University of Coimbra, Department of Informatics Engineering, 3030-290, Coimbra, Portugal \\ ${ }^{2}$ INESC Porto, 4200-465, Porto, Portugal
}

\begin{abstract}
The emerging of Quality of Service (QoS)-enabled broadband wireless access technologies brings internetworking facilities by allowing the development of systems with high speed communications as well as service differentiation and bandwidth guarantees for communication sessions. The end-to-end QoS control in heterogeneous environments, where networks may be composed of links with different bandwidth capacities and may support several QoS models, is still an open issue. This paper proposes a QoS control approach to guarantee the quality level of sessions crossing Worldwide Interoperability for Microwave Access (WiMAX) systems, independently of the QoS models or bandwidth capacities supported inside a WiMAX network as well as supported by a neighbour network. The proposed scheme is based on the coordination of resource allocation control with QoS mapping and adaptation mechanisms, which assures the quality level control of sessions over heterogeneous environments. Simulation results show the benefits of the proposed solution in reducing the session blocking probability and in optimizing the usage of network resources, while keeping sessions with an acceptable quality level during congestion periods.
\end{abstract}

Index Terms - Broadband Wireless Access, WiMAX, QoS Mapping, QoS Adaptation, Wireless Resource Allocation.

\section{INTRODUCTION}

$\mathrm{T}$ HE increasing number of mobile devices together with the emergence of broadband wireless access systems allows fixed and mobile users in metropolitan area networks to access real-time communication sessions ubiquitously, such as push media, IPTV and scalable video streaming. In addition to wireless and internetworking facilities, QoS guarantees and high speed transmission for communication sessions are also expected to be assured in broadband wireless access systems.

The WiMAX system, an IEEE 802.16 standardized architecture for all-IP networks, is the most attractive solution to broadband access in next generation systems [1]. The WiMAX system provides service differentiation based on the combination of a set of communication service classes supported in both wired IP-based and wireless IEEE 802.16based links. In the former, network elements with standard IP QoS models, such as Differentiated Services (DiffServ) [2] and Integrated Services (IntServ) [3], can be configured to guarantee QoS support for sessions crossing wired links. In the latter, several IEEE 802.16 QoS services are defined to provide service differentiation in wireless interfaces [4].

QoS models supported by WiMAX systems in wireless and wired elements, offer network services with different forwarding behaviours for packets, including performance metrics, such as bandwidth guarantee, tolerance to loss, delay and jitter. Therefore, sessions with similar QoS requirements must be mapped into appropriated wired and wireless service classes. With this goal in mind, it is required the use of a translation mechanism to allow an efficient mapping of sessions crossing networks that implement different QoS models. However, static approaches for QoS mapping between session requirements and network service classes, or even guidelines for IP QoS mapping alone, are not sufficient to assure the quality level of sessions. This is mainly due to the emergence of new QoS-aware sessions and network service classes with different configurations. Regarding the latter, each operator can use different parameters to configure its network classes according to its business model or internetwork agreements.

Additionally, due to the existence of wired and wireless links with distinct capacities and the dynamic bandwidth behaviour of the resources assigned for each service class, QoS mapping procedures must be done in cooperation with QoS adaptation support. In congestion periods, QoS adaptation schemes contribute to improve the usage of network resources and to avoid the session blocking, while keeping those sessions with an acceptable quality level. Thus, the satisfaction of users is increased because the session degradation is, in general, less annoying than to have a session refused. For instance, if a session is mapped into an overloaded class, a QoS adaptation scheme must be triggered to adapt the session to the current network conditions and to avoid the session blocking. This procedure can be done by requesting the re-mapping of the session to a different service class or by controlling the quality level of the session by dropping and adding low priority flows of a scalable session.

In addition to the QoS mapping and QoS adaptation mechanisms, a WiMAX-aware resource allocation scheme is essential to provide the control of network resources in wired and wireless service classes and to allow the configuration of the bandwidth required for each session in the selected classes. Summing up, the QoS control in WiMAX networks needs to be done by coordinating QoS mapping and QoS adaptation mechanisms with wired and wireless resource allocation controllers. This coordination aims to provide the

\footnotetext{
$\dagger$ This work was done in the context of the Q3M project, sponsored by DoCoMo Euro-Labs. At that time, Paulo Mendes was working at DoCoMo Euro-Labs.
} 
dynamic mapping between the session QoS requirements and the available service classes as well as this scheme minimizes the session blocking probability and optimizes the usage of resources in WiMAX systems composed either by wired and wireless QoS elements.

This paper presents the WiMAX Resource Allocation Control (WiRA) approach to provide QoS control for sessions crossing WiMAX systems, where it extends our previous work $[5,6]$ by integrating the benefits of QoS mapping and QoS adaptation schemes with WiMAX resource allocation control. This goal is achieved through the cooperation between QoS mapping, QoS adaptation and WiMAX resource allocation controllers. Both upstream and downstream QoS control operations are supported independently of the underlying QoS models and link capacities in WiMAX systems and in neighbour networks. The system flexibility is increased by using open interfaces with existing standards and solutions as well as by avoiding the installation of proprietary modules in end-hosts. Moreover, operational costs are expected to be reduced by decreasing the intelligence in the central wireless element, named Base Station (BS), and by avoiding QoS signalling exchanges in the wireless link. Simulation results present the benefits of this proposal in reducing the session blocking and in optimizing the usage of network resources when WiRA QoS adaptation controller is enabled and disabled. Furthermore, from the user point of view, WiRA aims to keep sessions with an acceptable quality level in a congestion period.

The remainder of this paper is organized as follows. Section 2 presents relevant related work. An overview of WiRA is described in Section 3. Section 4 introduces the WiRA performance evaluation. Finally, conclusions and future work are summarized in Section 5.

\section{RELATED WORK}

QoS control in WiMAX networks has been addressed by existing proposals. Among them, Carneiro et al [7] proposes a solution to support resource reservations in WiMAX wireless and wired links by using specific modules placed in network elements. However, the coordination of the proposed modules is performed by using Simple Network Management Protocol (SNMP) messages, which are sent in the wireless link through the second management connection. The use of the second management connection can raise performance issues, because the second connection is appropriated for delay tolerant traffic. Since many other IP protocol related messages share the same connection, the level of QoS provision will be affected [8]. In addition, this solution does not explain how the QoS mapping control of sessions, between wired and wireless QoS models, is accomplished.

An alternative solution presents a method for realizing dynamic QoS control in WiMAX systems [9, 10]. This solution defines a signalling approach to establish a service channel and a Media Gateway (MG) agent which makes awareness of the type of a session requested by a user. The claim of the solution is to establish a service channel from the MG to the user and, then, from the user to the base station. The service channel establishment takes into consideration the bandwidth requested by the user. This solution increases the system complexity by requiring the installation of extra modules in end-hosts. Furthermore, this approach does not provide the mapping of sessions between wired and wireless QoS models as well as the session adaptation to the current network conditions.

In what concerns QoS mapping schemes, the QoS Gateway (QoSGW) [11] is a centralized solution which acts as a QoS mediator between applications/devices and the underlying network QoS infrastructure. The QoSGW allows the mapping between the session requirements and the service classes supported by different QoS models. The main drawback of QoSGW resides is the fact that it requires the installation of proprietary modules in end-hosts, reducing thus the system flexibility and increasing QoS-related signalling in wireless interfaces. Another mapping solution also aims to guarantee QoS support for sessions between networks with different QoS models [12]. However, the proposed solution uses a QoS mapping scheme, where the end-to-end quality level of the sessions is not guaranteed in congestion periods, because the current conditions of the selected is not taking into account.

Another mapping scheme proposes a solution which integrates the support of QoS models, such as DiffServ and IEEE 802.16, and QoS static mapping strategies [13, 14]. The QoS control is achieved by implementing extra modules in all network elements of a system, including end-systems, and by using the Resource Reservation Protocol (RSVP) [15]. The RSVP messages can be sent in the wireless links through either, the primary or the second management connection. In the first case, the RSVP messages cross the wireless links encapsulated into DSA/DSC/DSD standard messages, contributing thus to increase the signalling overhead. In the second case, the QoS control procedures can be delayed due to the queuing characteristics of the channel. Moreover, the static QoS mapping allows only the mapping from DiffServ/IntServ to IEEE 802.16 QoS model (unidirectional mapping).

The analysis of related work has shown that none of the proposals satisfy all the requirements to provide a dynamic QoS mapping, QoS adaptation and resource allocation control for communication sessions crossing WiMAX systems. Most of the approaches do not adapt the session to the current network conditions to avoid the session blocking. In addition, existing solutions require extra QoS signalling in the wireless links and the installation of proprietary modules the end-hosts. Thus, the system flexibility and operational costs are negatively affected.

To overcome the above challenges and to provide QoS control for sessions between wired IP-based and IEEE 802.16based service classes in a WiMAX system, the University of Coimbra is working with DoCoMo Euro-Labs in the WiRA proposal, which will be presented in the next section. 


\section{WiMAX RESOURCE AlLOCATION CONTROL (WIRA)}

The WiRA QoS control solution in WiMAX networks is achieved by integrating QoS mapping, adaptation and WiMAX resource allocation control. The cooperation between these mechanisms provides QoS guarantees for sessions entering or leaving WiMAX systems, independently of the QoS models used in wired/wireless links or the current network conditions of the service classes. WiRA assumes that each session is described in a Session Object (SOBJ) and identified by a unique session identifier as proposed in the Next Step for Signalling (NSIS) framework [16]. In addition, each session can also be scalable and composed by a set of flows with different QoS requirements, priority and bit rate. The QoS parameters of each flow of a session are described in the QSPEC object [17], which includes performance values (e.g., bit rate, tolerance to loss, and delay jitter). These values can be quantitative (e.g., ms or kb/s) or qualitative (e.g., low, medium or high).

The SOBJ also includes information about the location of the user (named Subscriber Station (SS)) which requested the session, the IP address of the sender and the reservation direction, such as uplink or downlink direction in the WiMAX network. Besides the QoS control information described in the SOBJ/QSPEC, operators are responsible to export information about wired and wireless classes, including performance metrics and the per-class available bandwidth. Exporting theses QoS information, operators also keep their network infra-structure opaque. The generic definition of a SOBJ allows the use of any signalling protocol to transport the session QoS control information, such the QoS NSIS Signalling Layer Protocol (QoS NSLP) [18], the Session Initiation Protocol (SIP) [19] or RSVP. This control information can then be used by QoS mapping, QoS adaptation and resource allocation controllers to allow the session establishment with QoS support inside or between systems with different QoS models.

\section{A. WiRA Agents}

The WiRA QoS control solution is supported by agents distributed along a WiMAX network as illustrated in Fig. 1. On the one hand, WiRA-Stateful (WiRA-SF) agents are located in the ingress/edge wired element, called Connectivity Service Network (CSN), being responsible for the main WiRA control functions, such as admission control, session mapping and signalling initialization. The WiRA-SF agent implements all functionalities (and complexity) supported by the WiRA solution. WiRA minimizes the system complexity and operational costs by pushing the intelligence to the network edges. On another hand, WiRA-Stateless (WiRA-SL) agents are placed in the remainder WiMAX network elements (Access Service Network - Gateway (ASN-GW) and BS) and implement lightweight resource control procedures to configure the wired QoS resources and the wireless MAC classifier. Subscriber Stations (SS) do not use additional modules, which also improve the system flexibility.
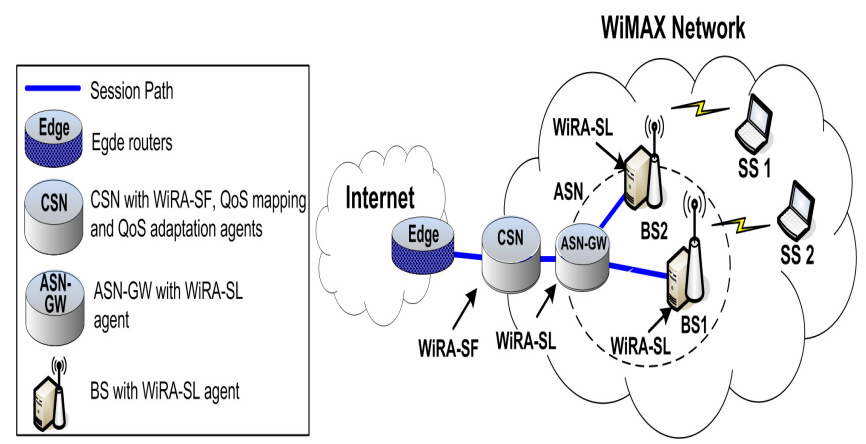

Fig. 1. WiRA Agents on the WiMAX network

When a session is leaving a WiMAX network, WiRA-SF performs the mapping (or adaptation) according to the available resources in inter-network classes or based on internetwork agreements.

\section{B. WiRA Interfaces}

WiRA implements open interfaces to allow a cross-layer interaction with existing protocols or mechanisms and an easy deployment in heterogeneous systems. The WiRA interfaces are the following:

- Application Interface (AI): used by WiRA to interact with external applications or mechanisms in order to receive QoS control requests for a session. The requester must provide the $\mathrm{SOBJ}$ and the reservation direction (uplink or downlink). This interface is also used by WiRA agents to trigger the requester application indicating the result of the requested operation, such as successful or not;

- Wired Resource Control Interface (WdRCI): used by WiRA to interact with QoS control plane elements to configure the resources required for sessions in wired links. For instance, the resource reservation is performed by the configuration of a QoS packet scheduler, such as Weighted Fair Queuing (WFQ) [20] or Class Based Queue (CBQ) [21]. Moreover, WiRA configures policies in order to mark incoming packets into a service class;

- Wireless Resource Control Interface (WrRCI): used by WiRA to interact with the IEEE 802.11 MAC layer to configure the MAC classifier. In addition, WiRA reserves resources in IEEE 802.16 wireless links by requesting the MAC layer to establish a transport connection with QoS guarantees for each flow of the session;

- Transport/routing Control Interfaces (TCI): used by WiRA to interact with the WiMAX ASN Transport Signalling Protocol (WATSP) [22] to transport QoS control information between WiRA agents. In addition, WiRA uses this interface to retrieve the outgoing interface associated with agents or users from local unicast routing tables. 


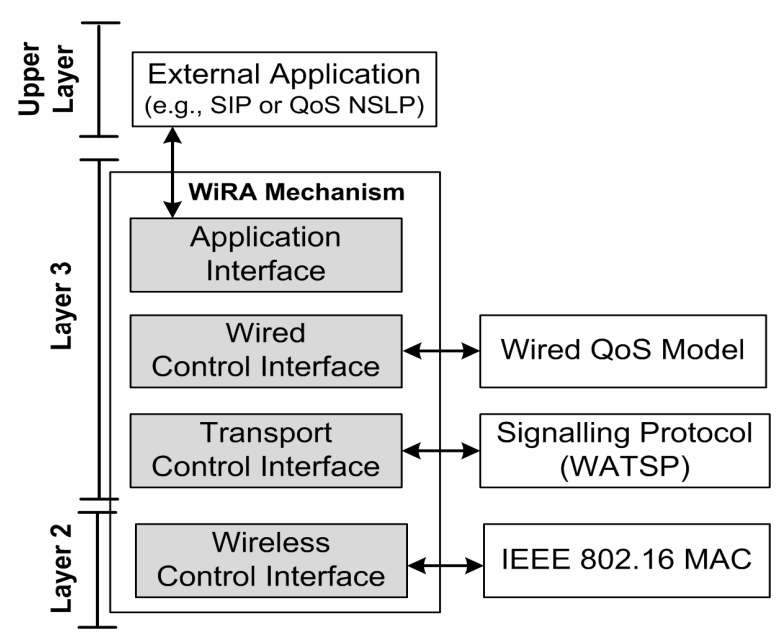

Fig. 2. Interfaces supported by WiRA agents.

Fig. 2 shows the interfaces supported by WiRA agents. A WiRA-SF agent supports almost all interfaces (it does not implement the WrRCI interface, because WiRA-SF agents do not configure wireless network elements). On the other hand, a WiRA-SL agent, located in the BS, supports the WdRCI, WrRCI and TCI interfaces, since it configures wired and wireless network elements. Finally, a WiRA-SL agent in internal WiMAX network elements (ASN-GW) only supports WdRCI and TCI interfaces, since it is only designed to configure the local wired network element and to propagate WATSP QoS messages.

\section{WiRA State}

In order to provide QoS control procedures, WiRA supports three databases to keep state about current sessions and network resources as follows:

- $\quad$ Wired Reservation Database (WdRD): this database keeps per-outgoing interface information about wired network resources, such delay, loss, jitter and available bandwidth associated with each class;

- Wireless Reservation Database (WrRD): this database is used by the WiRA-SF agent to control the wireless link utilization. Similarly to WdRD, the WrRD database keeps information about service classes, their QoS characteristics and available resources, for each BS and in both directions (downlink and uplink);

- Session Database (SD): this database is used to control current sessions in WiMAX networks, storing information about the resources associated with each session. This way, the SD database keeps the session identifier and flow identifier(s) as well as the service class, BS, Connection Identifier (CID) and bit rate assigned for each flow of a session.

The information stored in these databases enables WiRA to control QoS resources in the CSN-to-SS data path.

\section{WiRA Functionalities}

The WiRA functionalities are performed by using QoS mapping, QoS adaptation and resource allocation control mechanisms. The main functionality of each mechanism will be described in this section.

\section{1) WiRA Mapping and Adaptation}

The WiRA QoS Mapping and QoS Adaptation mechanisms are used to map the session requirements into available classes and to adapt the session quality level in congestion periods respectively. The mapping mechanism compares, one by one, the QoS parameters requested for each flow of the session (collected in the QSPEC) and the list of available service classes in the wired and/or wireless links. Then, it chooses the suitable service class for the requested session based on the perfect, sub-perfect or hybrid match methods. After the service class selection procedures, the mapping mechanism triggers the resource allocation controller to reserve resources for each flow of a session in the selected class. The QoS mapping is based on three methods are as follows:

- Perfect Match: the perfect match is assumed to be the preferred mapping method and supports the full QoS requirements and bandwidth committed for all flows of a session. In an unsuccessful case, such as, when the preferred network class has not enough available bandwidth to assure at least the minimal packet loss rate of the session, the QoS adaptation is triggered;

- Sub-perfect Match: maps all flows of a session to a network class that supports QoS parameters different from the ones described in the SOBJ. This method avoids the session blocking and the packet reordering, since all flows are mapped to another class. The sub-perfect method can be used in periods of congestion of the preferred class and when the session full rate is the major QoS requirement;

- Hybrid Match: assures the allocation of, at least, flows of a session with high priority into the preferred class. The remainder flows are mapped to a less suitable class. The hybrid method can be used when the packet re-ordering is not crucial. For instance, it can be appropriated for scheduled video and audio sessions, where it is more important to ensure an intelligible audio flow than a perfect video.

When the mapping process is not optimal, for instance due to a selection of an overloaded service class, the adaptation mechanism is triggered. The session is blocked only if a misplaced service class cannot guarantee the minimal QoS requirements desired for each flow of the session.

The quality level of sessions can be adapted by dropping or adding flows, by taking their priority into account as a way to reduce the impact in the session quality level. When the maximum bandwidth of the preferred class cannot assure the QoS committed for a low priority flow, this flow is removed from the outgoing interface and classified as sleeping state by the WiRA QoS adaptation mechanism. Sleeping flows are awaked when the network capability becomes available again and the session full rate can be supported. On the other hand, 
the re-mapping adaptation method requests the mapping of the session to another class (using the sub-perfect or hybrid mapping methods as explained before).

The process to decide which QoS mapping and adaptation methods must be used can be based on static or dynamic configurations. For instance, the mapping/adaptation system can be configured by the provider according to its business model, or on-demand by an external protocol.

\section{2) WiRA Resource Allocation}

The WiRA resource allocation mechanism aims to reserve resources and to configure schedulers for each session in the wired and wireless elements along the WiMAX network. In the former, the network resource allocation is performed by using the WdRCI interface. The reservations are accomplished by (i) configuring wired QoS schedulers (in DiffServ or IntServ enabled networks) and (ii) requesting the wirelessbased MAC layer to establish a new transport connection towards the SS with the requested QoS parameters. The packet marking is controlled by configuring QoS policies to assure that packets are properly classified into the selected network service classes.

\section{E. WiRA signalling}

WiRA uses the WATSP protocol to exchange control information between WiRA agents. This is done by using the RR-Request and the RR-Response messages. This set of WATSP signalling messages was extended by WiRA to carry QoS information needed to support the WiRA functionalities. Based on additional QoS information transported in WATSP messages, WiRA agents along the WiMAX network are triggered and execute their functions.

\section{F. WiRA Illustration}

This section describes the operations carried out by WiRA to establish QoS-aware sessions in a WiMAX network in both downlink and uplink directions.

\section{1) Downlink Illustration}

Fig. 3 illustrates an example of WiRA downlink QoS operations in a WiMAX system to setup a session.

The downlink QoS control process is started when the receiver ( $S S 1)$, which is placed in the WiMAX network, wants to subscribe the session (Sess 1). It is assumed that a network agent, in the CSN element, is triggered after the resource reservation process from the source to the CSN agent (step 1). This network agent is also responsible for triggering the WiRA-SF agent in order to request QoS guarantees for the session towards SSI. After that, the WiRA-SF agent queries its databases in order to obtain information about the available resources along the WiMAX network (in wired and wireless elements), and starts the mapping control. The mapping controller compares, one by one, the QoS parameters requested for each flow of the session (described in the QSPEC) and the list of available classes in the wired and wireless links, and selects IP-based and IEEE 802.16 classes to be used for each flow of Sess 1 .

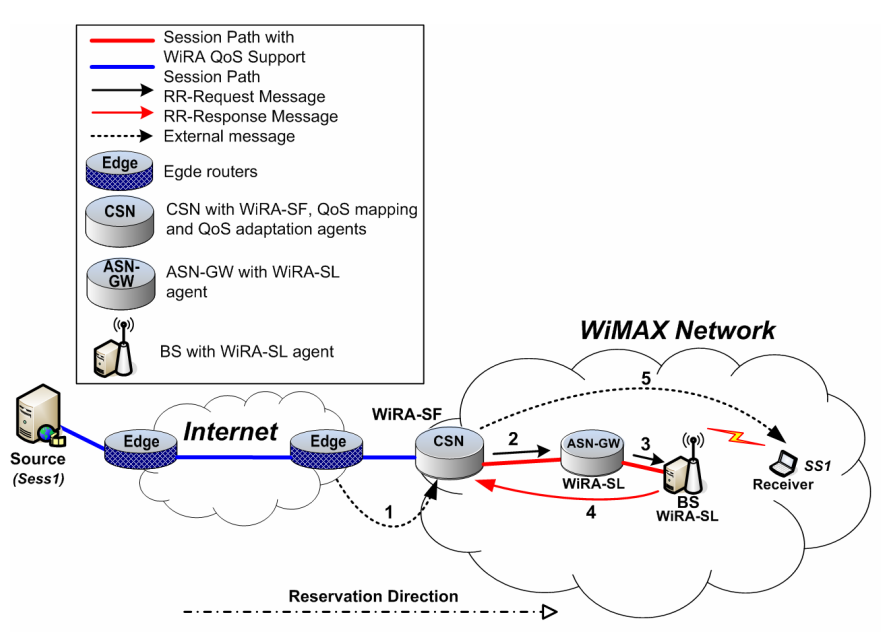

Fig. 3. Example of WiRA Downlink Operation

Note that when the selected service class (or classes) has not enough bandwidth to assure the minimal QoS committed rate for the session, the QoS adaptation process is triggered to adapt the session to the current network conditions. The adaptation mechanism aims to avoid the session blocking and to keep the session with an acceptable quality level.

After the QoS mapping control, the resource allocation process is triggered in order to reserve resources for Sess 1 in the preferred wired and wireless classes. This way, the WiRA-SF agent performs the local wired resource allocation process, and signals the WiRA-SL agent, located in the ASN$\mathrm{GW}$ element, by using a WATSP RR-Request message (step 2). Upon receiving the RR-Request message, the WiRA-SL agent accomplishes the wired resource allocation operation for Sess 1, and signals the WiRA-SL agent in the BS (step 3). Then, the WiRA-SL agent, in the BS, performs the wireless resource allocation process, and notifies the WiRA-SF, by using a WATSP RR-Response message (step 4), about the state of operation (in this case, a "successful" operation).

Finally, WiRA-SF updates its databases and informs the network agent about the success of the operation. The requester is responsible for informing the user application that the session was established with QoS support (e.g., by using a SIP message) (step 5). If, in any moment of the downlink operation, it is not possible to conclude the resource allocation process, WiRA releases the reserved resources, creates a $R R$ Response message with an error code. The RR-Response is sent through the reverse path, from the CSN to other WiRA agents, to release resources associated with that session as well as to notify the WiRA-SF agent about the lack of network resources.

\section{2) Uplink Illustration}

The uplink QoS control is requested when a receiver wants to subscribe the session Sess 2 offered by a source placed in the WiMAX network as presented in Fig. 4. The uplink process ensures resources for the session from the source to the CSN. It is assumed that a network agent in the CSN receives the user's request and triggers WiRA to start the reservation process (step 1). 


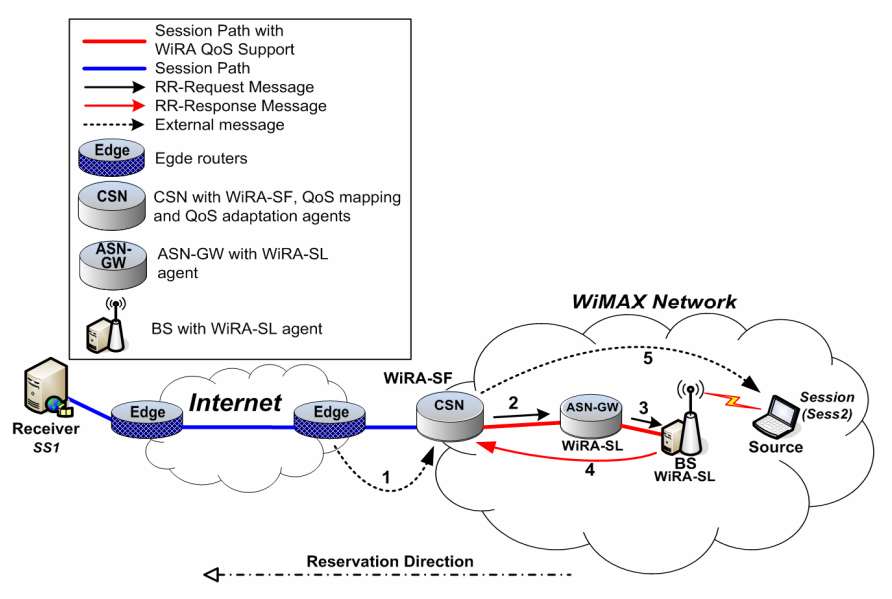

Fig. 4. Example of WiRA Uplink Operation

Upon receiving a triggering and the SOBJ, WiRA-SF is activated to perform QoS control for the session Sess 2 in the WiMAX network (in the upstream direction). Hence, the WiRA-SF agent retrieves, in its databases, information about the available network resources in wired and wireless links and starts the mapping control process.

After the execution of the QoS mapping process, WiRA-SF signals WiRA-SL agents along the WiMAX network to reserve resources for the session (step 2 and step 3). The WiRA-SL agent, in the ASN-GW, performs the resource allocation in the outgoing interface from the ASN-GW to the CSN as well as the WiRA-SL agent, in the BS, requests the establishment of a QoS-aware transport connection from the source to the BS and executes the resource reservation in the outgoing interface from the BS to the ASN-GW.

After the configuration of QoS paths associated with Sess2 in wireless and wired links, the WiRA-SL agent, in the BS, signals the WiRA-SF to inform it about the success of the uplink operation (step 4). Upon receiving the confirmation, WiRA-SF updates its databases and informs the requester about the success of operation, allowing the latter to notify the external source (step 5) and to allow the resource reservation process in the remainder path towards the receiver.

\section{Performance Evaluation}

The impact of WiRA to provide QoS control in WiMAX networks was evaluated through simulations. The objectives of the WiRA evaluation are twofold: ( $i$ ) analyze the amount of blocked session with and without WiRA adaptation control; (ii) analyze the usages of network resources in IEEE 802.16 service classes when WiRA is configured with different adaptation profiles.

The experiments were carried out by using the Network Simulator 2 (NS-2) [23]. The network topology is composed by four WiMAX network elements, CSN, ASN-GW, BS and SS, operating in WiMAX Pont-to-Multipoint (PMP) mode. The packet differentiation in wired links is accomplished by the DiffServ model (CSN -> ASN-GW -> BS), where three service classes were implemented as follows: Assured Forwarding (AF), Expedited Forwarding (EF) and Best-Effort (BE). Each service class is policed by a physical queue that is served based on the WFQ discipline. The packet differentiation in wireless links is performed by the IEEE 802.16 QoS model, where four classes were defined according the IEEE 802.16 standard as follows: Unsolicited Grant Service (UGS), Real Time Polling Service (rtPS), Non Real Time Polling Service (nrtPS) and BE.

In order to simplify the experiments, only downstream QoS control operations were analysed. Since packets were mapped from the wired service classes to the wireless service classes in the BS, all measurements were carried out in the BS. The capacities of the CNS-to-ASN-GW and ASN-GW-to-BS wired links are of $100 \mathrm{Mb} / \mathrm{s}$. Moreover, the propagation delay in the wired part is of $1 \mathrm{~ms}$. The uplink and downlink capacities of the wireless links are of $25 \mathrm{Mb} / \mathrm{s}$ and $50 \mathrm{Mb} / \mathrm{s}$ respectively. To avoid service class starvation, the network resources used by each class must not exceed $25 \%$ of the total bandwidth capacity of a link.

Additionally, the WiRA evaluation uses metrics of realtime sessions that are based on well-know scalable CODECS, such as Moving Picture Experts Group version 4 (MPEG4) [24]. Each scalable session is composed by a set of flows with well-defined priorities and exponential rates, where the adaptation process must be done taking into account the importance of each flow. Although the WiRA approach can handle any number of flows, the experiments will be based on sessions with three, four and five flows. This amount of flows provides a good trade-off between quality and bandwidth, and additional flows only provide marginal improvements [25]. The flows are sent with a constant bit rate of $32 \mathrm{~Kb} / \mathrm{s}, 64 \mathrm{~Kb} / \mathrm{s}$, $128 \mathrm{~Kb} / \mathrm{s}, 256 \mathrm{~Kb} / \mathrm{s}$ and $512 \mathrm{~Kb} / \mathrm{s}$, starting from the most important to the least important one respectively (from Flow 1 to Flow 5).

The simulations were performed with 1,000 sessions that were generated following a Poisson distribution. The session duration interval varies between $10 \mathrm{~s}$ and $30 \mathrm{~s}$. The duration interval times were chosen based on the duration of real traces used to evaluate the quality level of real videos [26]. The simulation time is of $100 \mathrm{~s}$, considering the duration of the session. A random function is responsible for generating the QSPEC object of each flow.

All experiments were carried out under the same simulation environment, and the difference between them resides in the QoS adaptation profile configured in each test. The following four main adaptation profiles are used to evaluate the WiRA QoS proposal: The $N_{-} A D P$ profile, in which no QoS adaptation method is used. In this case, the session is blocked if the complete set of QoS requirements cannot be assured in the preferred class. The ADP_Drop profile, in which session quality is controlled by dropping and adding low priority flows. The $A D P_{-} H y b$ profile, in which flows with high priority are mapped to the preferred class and flows with lower priority are re-allocated to a less important class. In this profile, the session is blocked only if the full rate of high priority flows cannot be assured in the preferred service class. The ADP_Sub profile, in which all flows of a session are remapped to a less important class. In this case, the session is 
blocked if the full rate of the session cannot be assured in the misplaced class (multi-user sessions re-mapped to the other service classes).

Fig. 5 illustrates the amount of blocked sessions in the wireless classes during the simulation with all adaptation profiles. The results reveal that 118 sessions were blocked when the QoS adaptation mechanism is disabled, while 55, 92 and 102 sessions were refused when the ADP_Drop, $A D P \_H y b$ and $A D P \_S u b$ profiles were used respectively.

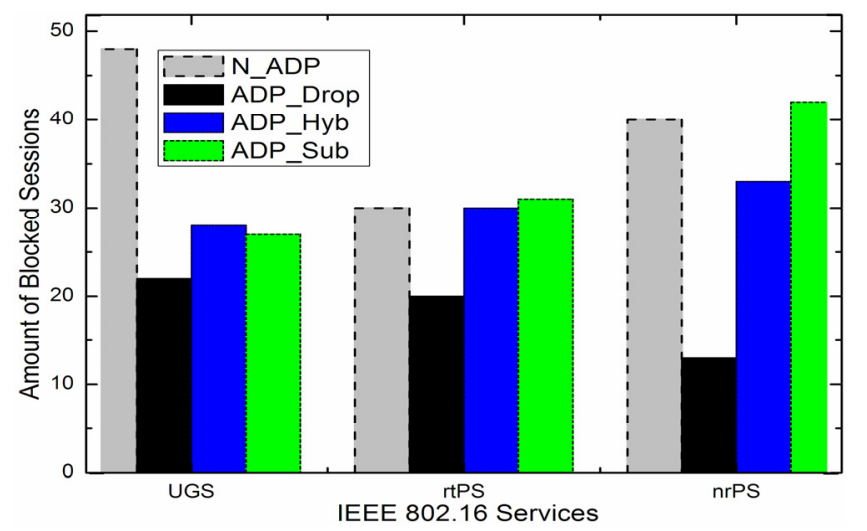

Fig. 5. Amount of blocked sessions in all adaptation profiles

When the ADP_Drop profile is configured, it minimized the number of blocked sessions in the WiMAX system by controlling the number of flows of a session. Compared with the $N_{-} A D P$ experiment, the $A D P \_D r o p$ profile reduced the number of refused sessions in $54 \%, 27 \%$ and $71 \%$ for sessions mapped in UGS, rtPS and $n r P S$ IEEE 802.16 classes respectively. In all experiments, the $A D P \_H y b$ profile reduced the number of blocked sessions compared with the $N_{-} A D P$ profile (by using resources allocated to other service classes). In addition, compared with the $N \_A D P$ profile, the $A D P \_S u b$ profile increased the number of blocked sessions in $3 \%$ for sessions mapped in $r t P S$ and $n r P S$ services. This occurs because misplaced sessions were using network resources allocated for sessions best suited in these less important service classes.

Fig. 6 illustrates the per-class usage of network resources for each IEEE 802.16 service class when the $N_{-} A D P$ profile is configured.

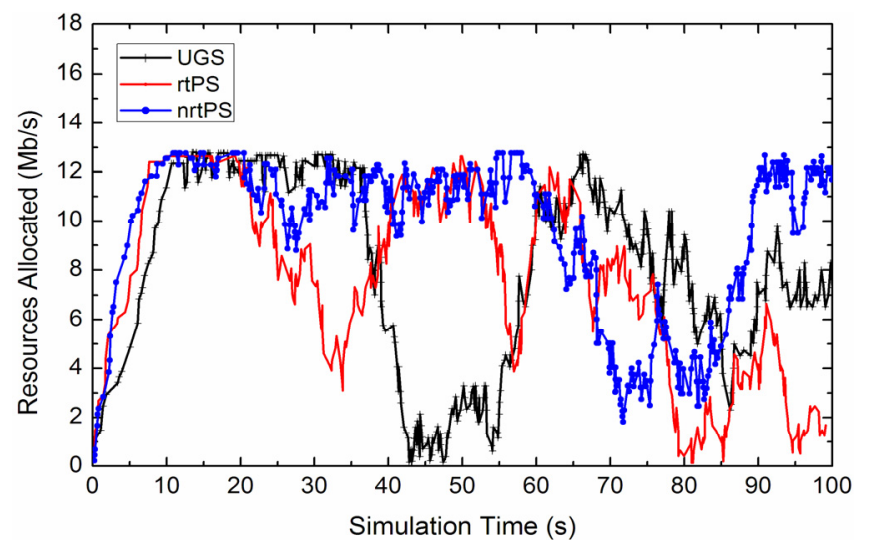

Fig. 5. Resources allocated for each IEEE 802.16 class when the system is configured with the $N_{-} A D P$ profile
The WiRA QoS adaptation control solution optimizes the usage of network resources in congestion periods of a service class, by using network resources available in other classes. Fig. 6 illustrates that between the instant $18 \mathrm{~s}$ and $39 \mathrm{~s}$ of the $r t P S$ class and between the instant $21 \mathrm{~s}$ and $31 \mathrm{~s}$ of the $n r P S$ class there are available resources that could be used to accommodate sessions refused in the $U G S$ class. When the WiRA QoS adaptation controller is enabled with the $A D P \_S u b$ and $A D P \_H y b$ profiles, the network resources available in the rtPS (depicted in Fig. 6) were used to accommodate 20 and 21 sessions respectively.

The main drawback of the adaptation $A D P_{-} S u b$ and $A D P_{-}$ $H y b$ methods is the fact that adapted/misplaced sessions consume network resources of other classes, which increases the call blocking of sessions best suited to those classes. For instance, 3 and 4 sessions best suited in the rtPS and accepted when the $N_{-} A D P$ profile is being used were refused when $A D P \_H y b$ and $A D P \_S u b$ are configured respectively.

\section{CONCLUSION AND FUtURE WORK}

This paper presents the WiRA proposal, which provides QoS support for sessions crossing WiMAX networks. This goal is accomplished through the cooperation between QoS mapping, QoS adaptation and WiMAX resource allocation controllers. Both upstream and downstream QoS control operations are supported by WiRA, independently of the underlying QoS models and link capacities in a WiMAX system and in a neighbour network. The WiRA flexibility is achieved by avoiding the time required by application level adaptation re-coding schemes and by avoiding the installation of proprietary modules in end-systems to perform QoS control procedures, such as resource reservation and QoS negotiation. With WiRA, the operational cost is expected to be reduced by decreasing the intelligence in the central wireless element (BS) and by minimizing QoS signalling exchanges in the wireless link. Furthermore, WiRA has interfaces, allowing operators to use signalling as well as intra and inter-network QoS controllers of their choice.

From the user point of view, the impact in the session quality level is reduced because the QoS adaptation process is performed taking into account the priority of each flow of a multi-user session as well as WiRA assures, at least, the minimal QoS parameters desired for the session even when a re-mapping procedure occurs. The simulation reveals that the WiRA QoS adaptation controller aims to reduce the amount of sessions blocked in congestion periods. In addition, it also aims to optimize the network resources allocated for service classes, by using resources available in other classes to accommodated misplaced sessions. For instance, when the system is configured to control the session quality level by dropping and adding low priorities flows of a session, the number of blocked sessions was reduced in 54\% compared to an experiment without WiRA adaptation control.

As future work, it is expected to analyse the benefits of the 
WiRA functionalities compared with other QoS control solutions. Furthermore, heuristics for the combination of all mapping and adaptation methods according to historical data and traffic patterns will also be investigated.

\section{ACKNOWLEDGMENT}

This work was done at the Laboratory of Communications and Telematics of the Faculty of Science and Technology of the University of Coimbra. It is supported by DoCoMo Euro-labs, by the Portuguese Ministry of Science, Technology and High Education, and by European Union FEDER - POSI (projects Q3M and SAPRA).

\section{REFERENCES}

[1] P. Iyer et al, "Al-IP network architecture for mobile WiMAX", Mobile WiMAX Symposium, Orlando, USA, March 2007.

[2] S. Blake et al, "An Architecture for Differentiated Service", IETF RFC 2475, December 1998.

[3] R. Braden et al, "Integrated Services in the Internet Architecture: an Overview", IETF RFC 1633, June 1994.

[4] C. Cicconetti et al, "Quality of Service Support in IEEE 802.16 Networks", IEEE Network, Vol. 20, N. 2, March 2006.

[5] E. Cerqueira et al, "QoS Mapping and Adaptation in Next Generation Networks", In Proc. of IEEE Workshop on Next Generation Service Platforms for Future Mobile Systems, Hiroshima, Japan, January 2007.

[6] E. Cerqueira et al, "QoS Mapping and Adaptation Control for Multi-user Sessions over Heterogeneous Wireless Networks", In Proc. of ACM International Mobile Multimedia Communications Conference, Nafpaktos, Greece, August 2007.

[7] G. Carneiro et al, "The DAIDALOS Architecture for QoS over Heterogeneous Wireless Networks", In Proc. of IST Mobile Communications Summit, Dresden, Germany, June 2005.

[8] IEEE 802.16-2004 Specification, "802.16-2004 IEEE Standard for Local and Metropolitan Area Networks, Part 16: Air Interface for Fixed Broadband Wireless Access Systems", Technical Report, October 2004.

[9] X. Zizhen et al, "Dynamic QoS Realizing Method in WiMAX Systems", Application Patent, N. PCT/CN2006/000950, November 2006.

[10] X. Zizhen et al, "A Method for Realizing the Dynamic QoS in WiMAX System", Application Patent, N. PCT/CN2006/000943, November 2006.

[11] M. El-Gendy et al, "Paving the first mile for QoS-dependent Applications and Appliances", In Proc. of IEEE International Workshop on QoS, Montreal, Canada, June 2004.

[12] M. Ruy et al, "QoS class mapping over heterogeneous networks using Application Service Map", In Proc. of IEEE International Conference on Networking, International Conference on Systems and International Conference on Mobile Communications and Learning Technologies, Washington, USA, April 2006.

[13] J. Chen et al, "Providing Integrated QoS Control for IEEE 802.16 broadband wireless access systems", In Proc. of IEEE Vehicular Technology Conference, Dallas, USA, September 2005.

[14] J. Chen et al, "An Integrated QoS Control Architecture for IEEE 802.16 Broadband Wireless Access Systems", In Proc. of IEEE Global Telecommunications Conference, St. Louis, USA, November 2005.

[15] R. Braden et al, "Resource Reservation Protocol (RSVP)", IETF RFC2205, September 1997.

[16] R. Hancock et al, "Next Steps in Signalling (NSIS): framework", IETF RFC 4080, June 2005.

[17] J. Ash et al, "QoS NSLP QSPEC Template", IETF Internet Draft (Working in progress), February 2008.

[18] J. Manner, G. Karagiannis, and A. McDonald, "NSLP for Quality-ofService Signaling", IETF Internet Draft (Working in progress), February 2008.

[19] J. Rosenberg et al, "SIP: Session Initiation Protocol", IETF RFC 3261, June 2002.

[20] M. Ashour and T. Le-Ngoc, "Performance Analysis of Weighted Fair Queues with Variable Service Rates", In Proc. of IEEE Global Telecommunications Conference, San Francisco, USA, December 2003.
[21] S. Floyd et al, "Link-sharing and Resource Management Models for Packet Networks", IEEE/ACM Transactions on Networking, Vol. 3, N. August 1995.

[22] WiMAX forum, "WiMAX End-to-End Network Systems Architecture" Technical Report, Stage 3, August 2006.

[23] VINT project, "Network Simulator 2", http://www.isi.edu/nsnam/ns/, accessed in November 2007.

[24] ISO-IEC/JTC1/SC297WG11, "Evaluation Methods and Procedures for July MPEG-4 Tests", Technical Report, 1996.

[25] K. Rose and S. Regunathan, "Toward optimality in scalable predictive coding", IEEE Transaction on Image Processing, Vol. 7, July 2001.

[26] Video Traces Research Group, "YUV 4:2:0 Video Sequences," http://trace.eas.asu.edu/yuv/qcif.html, Arizona State University, accessed in November 2007. 